\title{
Study of the Per OB2 star forming complex
}

\section{Structure and kinematics}

\author{
A. N. Belikov ${ }^{1}$, N. V. Kharchenko ${ }^{2}$, A. E. Piskunov ${ }^{1}$, E. Schilbach ${ }^{3}$, and R.-D. Scholz ${ }^{4}$ \\ 1 Institute of Astronomy of the Russian Acad. Sci., 48 Pyatnitskaya Str., Moscow 109017, Russia \\ e-mail: abelikov@inasan.rssi.ru, piskunov@inasan.rssi.ru \\ 2 Main Astronomical Observatory, 27 Academica Zabolotnogo Str., 03680 Kiev, Ukraine \\ e-mail: nkhar@mao.kiev. ua \\ 3 Astronomisches Rechen-Institut, Mönchhofstraße 12-14, 69120 Heidelberg, Germany \\ e-mail: elena@ari.uni-heidelberg.de \\ 4 Astrophysikalisches Institut Potsdam, An der Sternwarte 16, 14482 Potsdam, Germany
}

Received 3 October 2001 / Accepted 4 March 2002

\begin{abstract}
The study of a sky area with a 20 degree diameter centered on the nearby association Per OB2 has been carried out on the basis of the "Compiled Catalogue of Astronomical Data in the Per OB2 Field" presented in a previous paper. The catalogue contains about 30000 stars with accurate proper motions in the Hipparcos system as well as magnitudes supplemented with spectral classes and other relevant data. We applied the reduced proper motion technique to estimate individual distances for almost all catalogue stars. The distributions of reddening and interstellar extinction were determined on the basis of the spectral classification of stars in the area. Proper motions were corrected for differential rotation of the Galactic disk and parallactic motion effects and used for the evaluation of association membership. The analysis of a uniform subset of main sequence (MS) stars earlier than A7 - which is complete within $500 \mathrm{pc}$ - has clearly revealed the association as an area of enhanced density with an angular size of about $7^{\circ}$. In comparison to the previous results on Per OB2, we show that the presently determined area extends the association from its classical position towards the California nebula and the Auriga dark cloud. There are 1025 MS proper motion members residing in this region. The distance to the association centre derived from a kinematic calibration is found to be about 300 pc i.e., in perfect agreement with the Hipparcos data. The shape of the association is almost spherical, and its diameter found from stellar counts is about 40 pc. An examination of internal systematic motions from proper motion data has shown that there is no evidence of an expansion or a rotation of the association around the line of sight. The average tangential and spatial velocity vectors of Per OB2 with respect to the LSR are determined as $\left(V_{l}, V_{b}\right)=(+7.2 \pm 0.4,+2.0 \pm 0.3) \mathrm{km} \mathrm{s}^{-1}$ and $(U, V, W)=(+12.7 \pm 1.6,-3.0 \pm 0.6,-0.9 \pm 0.8) \mathrm{km} \mathrm{s}^{-1}$.
\end{abstract}

Key words. stars: distances - ISM: dust, extinction - stars: Hertzsprung-Russell (HR) and C-M diagrams - Galaxy: open clusters and associations: individual: Per OB2

\section{Introduction}

For about 50 years, stellar associations have been recognised to be the best representatives of regions with ongoing or just completed star formation. Many efforts were undertaken to obtain basic structural, kinematic and population parameters of these aggregates both in the Milky Way and in external galaxies (see Blaauw 1964 and Efremov 1989 for references to early works, or Sakhibov \& Smirnov 2000 for an example of the systematic study of extragalactic association populations).

Send offprint requests to: R. D. Scholz, e-mail: rdscholz@aip.de
The major problem in the study of nearby associations is the large area they occupy and the low apparent surface density which makes it difficult to separate members from rich fore/background field stars. Therefore, the most reliable method for the separation of the association from field stars is the kinematic selection based on proper motions. Compared to star clusters usually occupying rather limited sky regions, associations require proper motion surveys over fields covering a few hundred square degrees. In the pre-Hipparcos era this constrained the studies to bright stars having spectral classification. Therefore, the number of detected members did not exceed a few tens of stars. As a result, in spite of major efforts, detailed 
knowledge about the size, structure, kinematics, and population statistics was not obtained, especially for nearby OB associations.

After the completion of the Hipparcos mission, the astronomical community was provided with a tool perfectly suited for the investigation of nearby associations. There are two catalogues (Hipparcos and Tycho-1) compiled directly from the results of the Hipparcos mission as well as several others related to them: ACT RC, TRC and Tycho-2 which make use of earlier epoch data from the Astrographic Catalogue. These data are the realisation of a single and homogeneous astrometric and photometric system over the whole sky, which can hardly be achieved with ground based observations.

The work of de Zeeuw et al. (1999) presents the Hipparcos census of 12 nearby associations located less than 650 pc from the Sun. The membership determination for these stellar groups is based on the kinematic convergent point and the "spaghetti" criteria. The new data and selection methods provide an improved membership determination which corrects and updates the pre-Hipparcos lists of B-, A-type members of these groups. Typically, 100 members per association were found by Zeeuw et al. (1999). This should be compared with a few tens of stars per association known before Hipparcos. Based on the highly accurate Hipparcos data (parallaxes and proper motions), the distances of the association were improved, and spatial motions were computed.

The study of de Zeeuw et al. (1999) is of fundamental importance for forthcoming investigations of nearby associations, but it does not exhaust the full potential of the catalogues related to Hipparcos. The Hipparcos catalogue itself is complete only for bright stars and does not provide sufficient statistics for a systematic study of populations in nearby associations which requires a completeness at $V>10 \mathrm{mag}$. The Tycho- 2 catalogue contains about twenty times more stars and is complete down to about four magnitudes fainter than the Hipparcos catalogue. On the northern sky there is a recently published deeper catalogue FONAC (Kislyuk et al. 1999) for stars listed in the Astrographic Catalogue. For the nearest associations, Tycho-2 and FONAC provide a completeness of $\mathrm{F}$ and $\mathrm{G}$ stars and can be used for the study of Pre-MS branches in their CMDs and LFs which are of a key importance in understanding the history of star formation and of the IMFs in these aggregates.

Per OB2 is one of the nearest OB associations that has been attracting the attention of many investigators (see de Zeeuw et al. 1999 for references). At a Galactic latitude of $b \approx-15^{\circ}$, the association is projected on a relatively poor stellar background which is an advantage for the membership determination. On the other hand, its kinematics do not differ considerably from the kinematics of field stars. This leads to uncertainties in the overall membership determination based on proper motions. For example, out of 17 classical pre-Hipparcos members, Hipparcos confirmed only about half of them (de Zeeuw et al. 1999). This underlines the importance of a system- atic study of the selection of association members with a higher reliability.

For this purpose, we compiled a catalogue (Belikov et al. 2002, referred hereafter as Paper I) containing the Per OB2 region with a diameter of 20 degrees centered at $(\alpha, \delta)_{J 2000}=3.87,34.4(l, b=160.53,-14.73$, see Fig. 1). The Compiled Catalogue (CC) is based on published astronomical data including Hipparcos, Tycho-2, and FONAC. It contains about 30000 stars, and is complete down to $V=11.6 \mathrm{mag}$. Heterogeneous data are reduced into homogeneous systems: e.g., the coordinates and proper motions are in the Hipparcos system, the photometry is in the Johnson system.

The region covered by the $\mathrm{CC}$ has a complex structure: it includes dark and dense clouds, young stellar groups and OB stars. Dust clouds distributed both within the association and between the association and the Sun (see e.g. Cernis 1993) screen different parts of the area studied. In Fig. 1 we show a map of the wider neighbourhood of the studied region for stars brighter than $V=11.6$ mag.

In the present study we aim to:

1. select a complete sample of Per OB2 members based on accurate proper motions corrected for systematic effects (differential Galactic rotation and parallactic motion);

2. study interstellar extinction in the area of the association based on accurate photometry and spectral classification data;

3. determine the structural parameters of Per OB2 (distance and size) and of its internal (expansion and/or rotation) and mean motion.

This paper has the following structure: in Sect. 2 we describe a procedure for the construction of a kinematically calibrated distance scale and its properties. Section 3 is devoted to the study of the distribution of interstellar extinction in the neighbourhood of Per OB2. In Sect. 4 we discuss the adopted algorithm of the membership determination and the resulting samples. Spatial structure and kinematics of the association are derived in Sects. 5 and 6 . In Sect. 7 we discuss and summarise the results.

\section{The reduced proper motion calibration with absolute magnitude and distance}

As the analysis in Paper I shows, the proper motion members of the Per OB2 association are distributed over the whole area covered by the $\mathrm{CC}$. At the average distance of $300 \mathrm{pc}$ adopted for the association (see Paper I and references therein), this leads to a total diameter of the association of at least $100 \mathrm{pc}$. Since both lengths are of the same order, a spatial (3-dimensional) approach to the study of the region seems to be mandatory.

Unfortunately, individual distances can be derived only for a small fraction of CC stars by conventional methods (e.g., from Hipparcos parallaxes or from a spectral class - absolute magnitude calibration). Therefore, we decided to use the reduced proper motion technique for the 


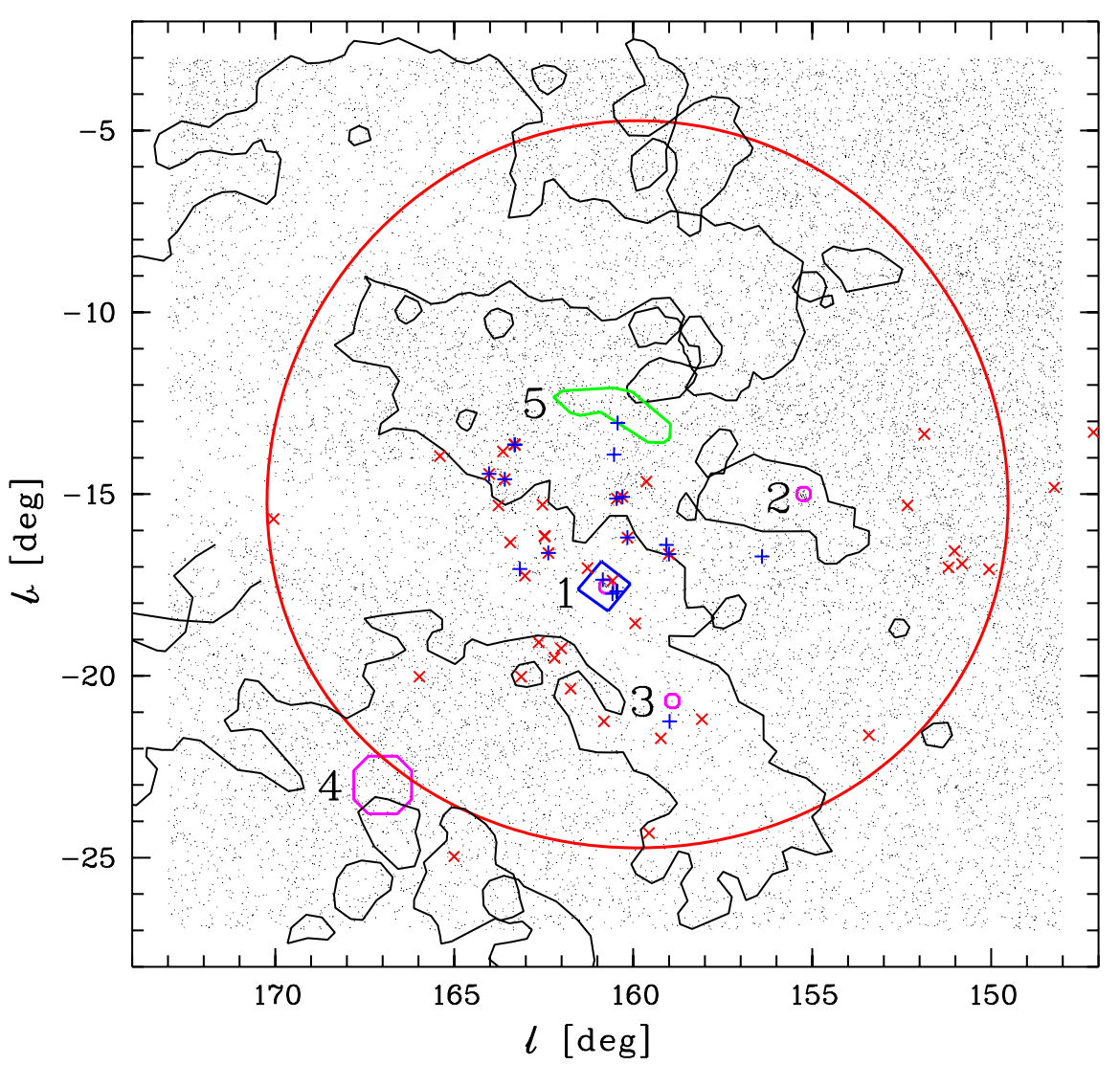

Fig. 1. A map of the area covered by the CC (shown by the large circle). Dots are stars from the Tycho2 catalogue, plus signs are Blaauw' (1952), and crosses are Hipparcos' (de Zeeuw et al. 1999) members of the Per OB2 association. The thin solid curve shows the CO-emission contours from Ungerechts \& Thaddeus (1987) survey. The small square is the field covered by the proper motion survey of Scholz et al. (1999) in the vicinity of IC 348. The grey symbols with numbers mark: 1 - the young cluster IC 348,2 - the open cluster NGC 1342, 3 - the position of the embedded cluster NGC 1333, 4 - the Pleiades open cluster, and 5 California emission nebula NGC 1499. distance evaluation (see e.g. Jones 1972; Chiu 1980). For each star, we computed the "reduced proper motion" $H_{V}$ from its magnitude $V$ and proper motion $\mu$ as

$$
H_{V}=V+5+5 \lg |\mu|
$$

where $\mu$ is in arcsec/yr. In order to calibrate the $H_{V}$-based distance scale, we derived relations between the absolute magnitudes of dwarf stars $^{1}$ of different spectral classes and the corresponding mean reduced proper motions. For dwarf stars with known spectral classes the average values $\bar{H}_{V}$ have been computed for each spectral group, with typically a few dozens of stars in each group, and compared with the absolute magnitudes $M_{V}^{\mathrm{SK}}$ from the tables of Schmidt-Kaler (1982). We have found that within the $\mathrm{CC}$ both values are in perfect correlation:

$M_{V}^{\mathrm{SK}}=(0.97 \pm 0.01) \bar{H}_{V}-(3.8 \pm 0.1)$.

We compared Schmidt-Kaler's absolute magnitudes and colours for luminosity class V with the Hipparcos (Hipp) spectral calibration of Houk et al. (1997), and found that both scales are in good agreement. The corresponding least-squares solutions for absolute magnitudes

$M_{V}^{\mathrm{SK}}=(1.01 \pm 0.02) M_{V}^{\mathrm{Hipp}}+(0.09 \pm 0.08)$,

and for intrinsic colours

$\underline{(B-V)_{0}^{\mathrm{SK}}=(1.02 \pm 0.02)(B-V)_{0}^{\mathrm{Hipp}}-(0.02 \pm 0.01),}$

${ }^{1}$ Formally, the kinematic calibration was also constructed for giants, but due to the low reliability of the spectroscopic distance calibration, they were not used in the evaluation of the structure and distance of the association. of B8-G8 dwarfs do not show any significant difference. We conclude that the Schmidt-Kaler calibration is in agreement with the Hipparcos scale. However, for later spectral types the calibrations differ significantly, and the corresponding distances could be considerably biased.

Taking into account Eq. (1), we can compute $M_{V}^{H_{V}}$ for each star. The corresponding distance scale can be obtained from

$\lg d=0.2\left(V-A_{V}-M_{V}^{H_{V}}\right)+1$,

and should be close to the Hipparcos system. For stars with known $M K$-classification, the comparison of $M K-$ and $H_{V}$-calibrated distances gives a kind of "internal" error of the calibration which depends mainly on kinematic inaccuracies (e.g., proper motion errors, neglecting of the random orientation of individual velocity vectors). For $d<600 \mathrm{pc}$, we found that the relative distance differences $\left(d^{M K}-d^{H_{V}}\right) / d^{M K}$ are randomly distributed with an average of $(1 \pm 2) \%$ and a standard deviation of $22 \%$. The "external" accuracy can be evaluated from the comparison with Hipparcos parallaxes. For a sample of 102 MS stars within $500 \mathrm{pc}$ and with $\varepsilon_{\pi}<5$ mas, $\varepsilon_{\mu}<5 \mathrm{mas} / \mathrm{y}, \pi>\varepsilon_{\pi}$, $\mu>\varepsilon_{\mu}$ we obtained the mean difference $\left(d^{\pi}-d^{H_{V}}\right) / d^{\pi}$ of $(-1 \pm 5) \%$ with a standard deviation of $52 \%$. In this case, the differences are caused by both parallax and kinematic errors. Therefore, we concluded that our kinematic calibration is accurate within 20 to $50 \%$.

In order to better understand the properties of the adopted distance indicator $d$, we have constructed a model of the studied population based on the Monte Carlo 


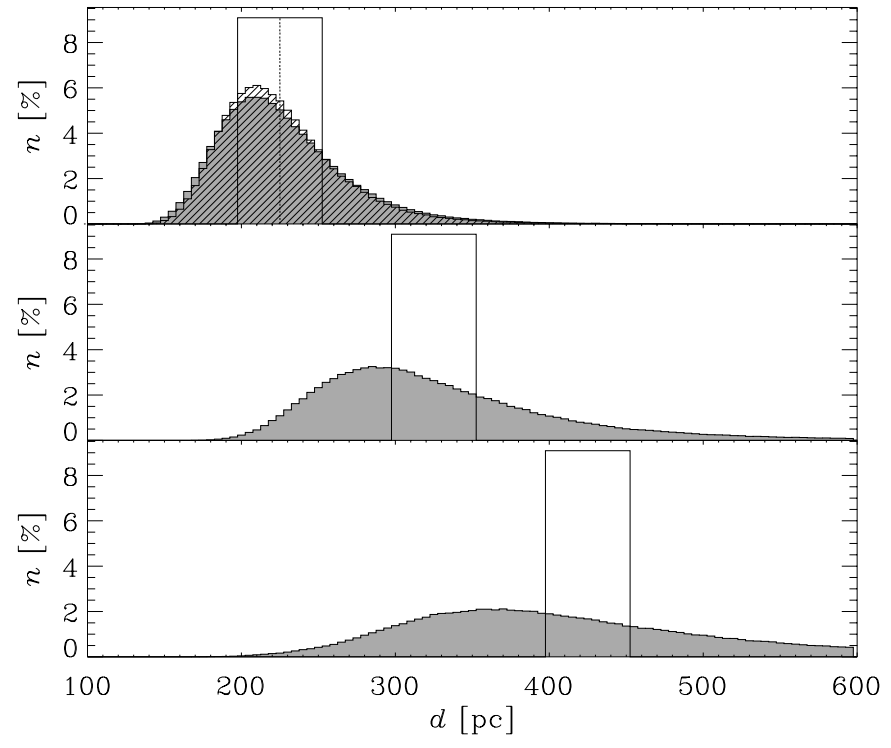

Fig. 2. Model distribution of $H_{V}$-calibrated distances (filled histogram) for rectangular initial profiles $(\Delta d=50 \mathrm{pc})$ located at different average distances $\left(d_{\mathrm{a}}\right)$ where $n$ is normalised number of stars within a distance range. Upper panel: $d_{\mathrm{a}}=225 \mathrm{pc}$. The hatched histogram corresponds to the profile with $\Delta d=$ $0.1 \mathrm{pc}$, located at the same distance. Middle panel: $d_{\mathrm{a}}=325 \mathrm{pc}$, bottom panel: $d_{\mathrm{a}}=425 \mathrm{pc}$.

method. The model describes a stellar group of finite size $\Delta d$, with a center located at the distance $d_{\mathrm{a}}$ from the Sun and predicts an observed distribution of stars as a function of $d$.

We assumed that the stars are of the same age and they are distributed along an appropriate isochrone $(\log t=$ 7.0) with the Salpeter initial mass function (IMF). The spatial distribution of the stars is approximated by a rectangular profile. The group has an average spatial motion with respect to the Local Standard of Rest (LSR) which is similar to that of the Per OB2 complex. We also assumed that the stars show a random internal motion with a dispersion of $1 \mathrm{~km} \mathrm{~s}^{-1}$. The observed photometric and proper motion errors, typical for the CC, were adopted for the model sample, too.

In Fig. 2 we show the model distribution of $\mathrm{H}_{V^{-}}$ calibrated distances $d$ for stellar groups with a size of $\Delta d=50 \mathrm{pc}$, located at different distances $\left(d_{\mathrm{a}}=\right.$ $225,325,425 \mathrm{pc})$. Two conclusion can be drawn: at larger distances the width of the distribution increases, and the maximum of the distribution systematically moves to smaller distances compared to the averaged distance assumed for the group (similar to the Lutz-Kelker bias in the determination of averaged distances from parallax measurements). The upper panel compares the distributions of two groups of different sizes: $\Delta d=0.1$ and 50 pc. As both groups produce $d$-distributions of the same width, we can conclude that the finite size of a group cannot be regarded as the primary reason for the distribution widening. The basic factors distorting the distance distributions are internal motion and errors in the kinematic data. Indeed, an internal velocity dispersion of $1 \mathrm{~km} \mathrm{~s}^{-1}$ leads to a proper motion dispersion of $1 \mathrm{mas} / \mathrm{yr}$ at a distance of about $200 \mathrm{pc}$ which, in turn, corresponds to a typical proper motion error of brighter stars in the CC.

In order to use the $H_{V}$-calibrated distances for the determination of the average distance to the association, we must introduce a correction for the bias described above. From model calculations we found that the average distance to the association $d_{\mathrm{a}}$ can be derived from a relation

$d_{\max } \approx k \times d_{\mathrm{a}}$,

where $d_{\max }$ is position of the maximum of the observed distribution and $k$ is a factor ranging within $0.82 \div 0.88$. This relation is valid for a distance of $d_{\mathrm{a}}=200 \div 600 \mathrm{pc}$ and for an association depth of $\Delta d=0.1 \div 200 \mathrm{pc}$.

\section{Interstellar extinction toward the Per OB2 complex}

As in any other star forming region, extinction in the Per OB2 area varies in the face-on plane as well as along the line of sight (see e.g. Černis 1993 and Ungerechts \& Thaddeus 1987). In this section we develop the appropriate tool for the reddening study, discuss the spatial distribution of the interstellar extinction towards the Per OB2 complex and provide the extinction corrections for the majority of stars of the CC.

According to the type of data available for the evaluation of the interstellar extinction, we divided stars from the $\mathrm{CC}$ into three groups:

1. 619 stars with known spectra and luminosity classes;

2. 5968 stars with known spectra but missing luminosity classes;

3. 19925 stars without information on spectra and luminosity classes but with proper motions available.

For all these stars $B, V$-magnitudes are available in the CC. To obtain the extinction parameters for stars of group 1, we used the following relations:

$E_{(B-V)}=(B-V)-(B-V)_{0}$,

$R_{V}=R_{0}+q E_{(B-V)}$,

$A_{V}=R_{V} E_{(B-V)}$,

$V_{0}-M_{V}=V-M_{V}-A_{V}$.

The intrinsic colours $(B-V)_{0}$ and absolute magnitudes $M_{V}$ were taken from the tables of Schmidt-Kaler (1982). For the values of $R_{0}$ and $q$ which are functions of temperature and luminosity class we used data given by Straižys (1977). As a result, the reddening $E_{(B-V)}$, extinction $A_{V}$, and distance modulus $V_{0}-M_{V}$ were obtained for 527 stars. For the remaining stars, no consistent solutions were found. Therefore, they were moved to group 3.

The missing luminosity classes for the stars in group 2 were derived by use of the reduced proper motion-colour diagram $\left(H_{V},(B-V)\right)$ where the domains of the main sequence and giant stars were identified by stars with known 


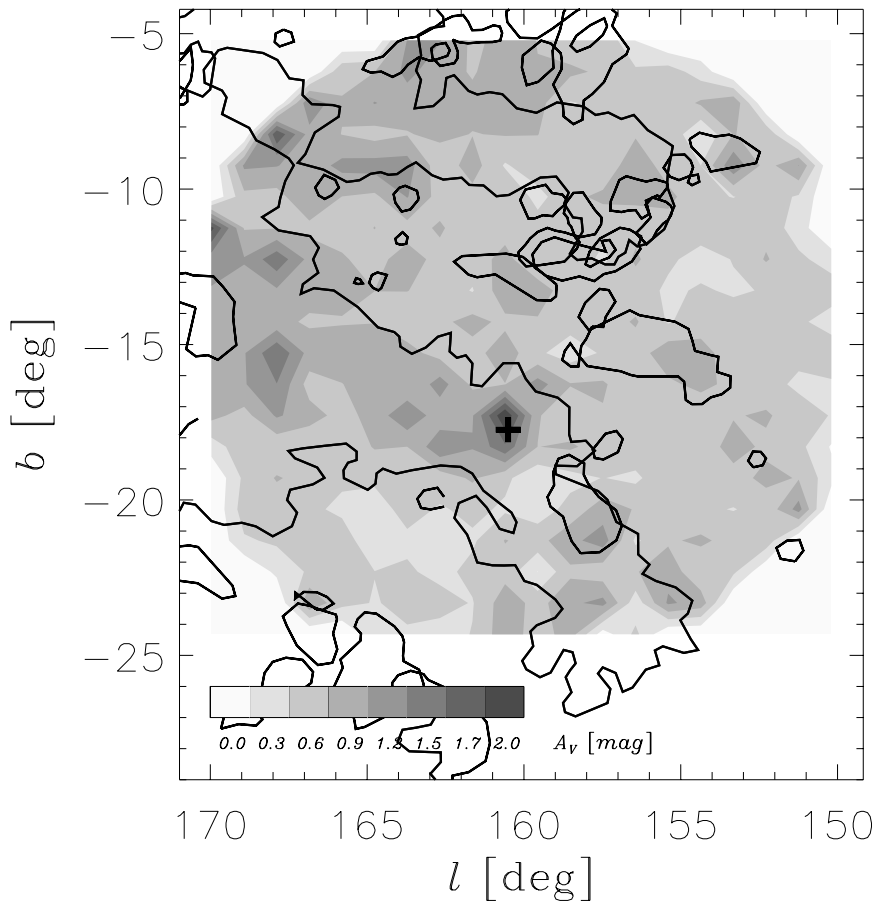

Fig. 3. The extinction map of the Per OB2 region based on the data of 2929 stars from groups 1 and 2. The extinction value is shown by gray colour according to the scale at the bottom of the figure. Solid curves represent the CO-emission contours from the survey by Ungerechts \& Thaddeus (1987). The plus sign marks the position of the young cluster IC 348 .

luminosity classes (group 1). Then, $A_{V}, E_{(B-V)}, R_{V}$ and $(m-M)_{0}$ were computed by the same procedure as described above. For 4850 stars, the solution was accepted, the remaining stars were moved to group 3.

For the determination of the reddening and extinction for stars from group 3, we made use of a map of the spatial distribution of the extinction in the region covered by the CC. The extinction map was constructed from the data of stars selected from groups 1 and 2. First, we excluded stars with poor photometry

$E_{(B-V)} / \varepsilon_{(B-V)}<1.0$,

where $\varepsilon_{(B-V)}$ is the $r m s$-error of the colour. We restricted the sample to main sequence stars since the intrinsic colour calibration for red giants is too uncertain. In Fig. 3 we show the extinction pattern based on 2929 reference stars selected from groups 1 and 2. A remarkable correlation of optical extinction and CO-emission intensity is obvious.

Individual extinction values $A_{V}$ for stars of group 3 were computed from Eq. (2) solved by an iterative procedure. The initial approximation of the distance $d$ was calculated with $A_{V}=0$. Then, the value of $A_{V}$ was derived as the average of the $A_{V}$-values of the 5 nearest reference stars used for the construction of the extinction map. The iteration was stopped when the difference between successive $d$ values was less than 1 per cent, or when the number

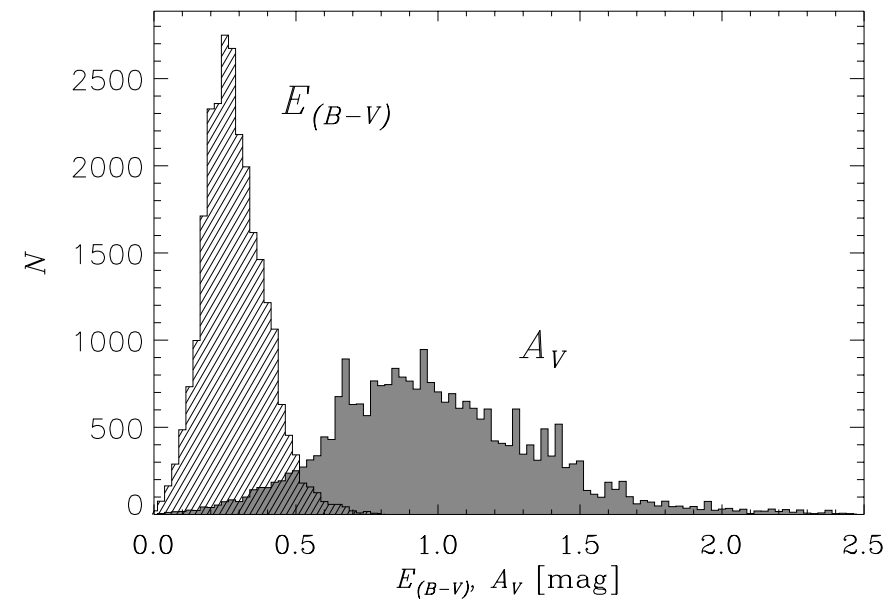

Fig. 4. Distribution of the CC stars with the reddening $E_{(B-V)}$ (hatched) and interstellar extinction $A_{V}$ (filled).

of iterations exceeded 20. In the latter case, this star was not considered further.

The distributions of $26380 \mathrm{CC}$ stars versus reddening $E_{(B-V)}$ and extinction $A_{V}$ are shown in Fig. 4. According to this plot, the typical reddening for the studied area is not very strong with $\bar{E}_{(B-V)}=0.31 \mathrm{mag}$, although the maximum reaches $1 \mathrm{mag}$. The typical extinction of $\bar{A}_{V}=1.0 \mathrm{mag}$ in the Per OB2 direction accumulated at a distance of $0.5 \mathrm{kpc}$, is higher than the average value $a_{0}=1.6 \mathrm{mag} / \mathrm{kpc}$ usually assumed for the Galactic plane. The estimated $E_{(B-V)}$ and $A_{V}$ values have been added to the actual $\mathrm{CC}$ version currently stored at the CDS.

Although almost all CC stars got distance and reddening values, only the results for main sequence stars with spectral classes earlier than G8 can be regarded as sufficiently reliable. These stars are flagged in the CC and used in our following study.

We compared the computed values of $A_{V}$ for 174 stars of groups 1, 2 and 33 stars of group 3 with published data (Seyfert et al. 1960; Černis 1990; Černis 1993). Average differences between the values derived in this paper and published absorption values are $0.04 \pm 0.01 \mathrm{mag}$ for groups 1, 2 and $0.06 \pm 0.04 \mathrm{mag}$ for the remaining stars, which indicates that a systematic bias is practically absent. The corresponding standard deviations of the differences are $0.19 \mathrm{mag}$ and $0.25 \mathrm{mag}$. Since the typical value of $A_{V}$ is $1.0 \mathrm{mag}$ (see Fig. 4), we conclude that our extinction values are accurate within $20 \%$ for the stars of groups 1, 2 and within $25 \%$ for the remaining CC stars.

\section{Association membership}

In Paper I, we used proper motions to compute probabilities of the belonging of stars to the Per OB2 association. Now, with individual distances for each CC star, we can check or even improve the membership determination.

Member selection based on kinematic criteria makes use of the fact, that stars belonging to a moving group (a cluster, an association, etc.) have practically the same 
spatial velocities. For a proper implementation of the kinematic approach, we must, therefore, take into account the influence of systematic motions on stellar kinematics.

The most prominent biases are the differential rotation of the Galactic disk and the Solar spatial motion. Stars of the CC occupy an area of the sky with a diameter of about 20 degrees, and the depth of the $\mathrm{CC}$ sample along the line of sight exceeds 500 pc. Thus, for stars located in the centre or at the borders of the region, respectively, the velocities due to parallactic motion or differential rotation differ by $\sim 3-4$ mas/yr in angular and $\sim 3-4 \mathrm{~km} \mathrm{~s}^{-1}$ in linear scales. The mean motion of the association as well as the peculiar internal motions are of the same order of magnitude, too. Therefore, independent of the kinematic parameter (proper motion, tangential, radial or spatial velocity) used for the calculation of membership probability, it has to be corrected for the effect of systematic motions.

In order to take into account both biases, we used data based on Hipparcos. The corrections were computed in a rectangular coordinate system $(X, Y, Z)$ centered on the Sun where the corresponding space velocity vector is $(U, V, W)$. The $X$ and $U$ axes point towards the Galactic anticenter $(l, b)=\left(180^{\circ}, 0^{\circ}\right), Y$ and $V$ are directed towards Galactic rotation $(l, b)=\left(90^{\circ}, 0^{\circ}\right)$, and $Z$ and $W$ towards the North Galactic Pole $\left(b=90^{\circ}\right)$. The Solar velocity with respect to the LSR in this system was adopted according to Dehnen \& Binney (1998) as $\left(U_{\odot}, V_{\odot}, W_{\odot}\right)=(-10.00 \pm 0.36,+5.23 \pm 0.62,+7.17 \pm$ $0.38) \mathrm{km} \mathrm{s}^{-1}$. Values of Oort constants were taken from Feast \& Whitelock (1997): $A=+14.82 \pm 0.84$, and $B=$ $-12.37 \pm 0.64 \mathrm{~km} \mathrm{~s}^{-1} / \mathrm{kpc}$.

The corresponding corrections of the proper motion $\delta \mu$ and radial velocity $\delta V_{R}$ were computed from the following equations:

$$
\begin{aligned}
& \delta \mu_{l^{*}}=\delta \mu_{l} \cos b=(A \cos 2 l+B) \cos b / \eta, \\
& \delta \mu_{b}=-A \sin 2 l \sin 2 b /(2 \eta), \\
& \delta V_{R}=A d \sin 2 l \cos ^{2} b, \\
& \delta \mu_{l^{*}}=\left(-U_{\odot} \sin l-V_{\odot} \cos l\right) /(\eta d), \\
& \delta \mu_{b}=\left(-U_{\odot} \cos l \sin b+V_{\odot} \sin l \sin b-W_{\odot} \cos b\right) /(\eta d), \\
& \delta V_{R}=U_{\odot} \cos l \cos b-V_{\odot} \sin l \cos b-W_{\odot} \sin b,
\end{aligned}
$$

where $\eta=4.74$. The corrections of tangential velocities were derived from $\delta \mu_{l^{*}, b}$ with

$V_{l, b}=\eta \cdot \mu_{l^{*}, b} \cdot d$.

$V_{l, b}$ is a component of tangential velocity in $l$ or $b$ direction in units of $\mathrm{km} \mathrm{s}^{-1}$ whereas proper motions $\mu_{l^{*}, b}$ and distance $d$ are given in units of mas/yr and $\mathrm{kpc}$, respectively. The first three relations in (4) refer to the effect of differential rotation, the remaining three deal with the effect of parallactic motion. The derived corrections were subtracted from the observations. Due to the proximity of the association, the corrections of the second order can be safely neglected (they are only significant for distances exceeding $1 \mathrm{kpc}$ ).

From the proper motions corrected for systematic motions, we re-computed $H_{V}$-values. Only for $1 \%$ of the
CC stars, $H_{V}$-values changed by more than $0.2 \mathrm{mag}$. We conclude that the calibration error arising due to neglecting systematic motions is comparable with the calibration accuracy (see Eq. (1)).

Although Per OB2 is not clearly separated from field stars in proper motion space, we were able to detect two association populations (A and C) in Paper I and calculate membership probabilities based on proper motions, respectively $P_{\mathrm{a}}$ and $P_{\mathrm{c}}$. We attributed the difference in average proper motions of the groups to their different location within the association, i.e., population $\mathrm{C}$ represents the part of the association near to the Sun, whereas population A consists of the rest of association members.

With individual distances of the stars available, we can compute membership probabilities $P_{\mathrm{t}}$ based on tangential velocities, describing the association as a whole. According to Eq. (2) of Paper I, we computed $P_{\mathrm{t}}$ for a star $i$ as

$P_{\mathrm{t}}^{i}=\exp \left\{-\left[\left(\frac{V_{l}^{i}-\bar{V}_{l}}{2 \varepsilon_{V_{l}}^{i}}\right)^{2}+\left(\frac{V_{b}^{i}-\bar{V}_{b}}{2 \varepsilon_{V_{b}}^{i}}\right)^{2}\right]\right\}$.

Here $V_{l}^{i}$ and $V_{b}^{i}$ are the components of the tangential velocity of a star $i$ computed from proper motions corrected for differential rotation and parallactic motion in accordance with Eqs. (4) and (5); $\varepsilon_{V_{l}}^{i}$ and $\varepsilon_{V_{b}}^{i}$ are the corresponding mean errors. The averaged tangential velocities $\bar{V}_{l}$ and $\bar{V}_{b}$ were found in a series of iterative steps. As an initial approximation, $\bar{V}_{l}$ and $\bar{V}_{b}$ were computed for a sample with the combined proper motion probability $P_{\mathrm{ac}}^{i}=\max \left\{P_{\mathrm{a}}^{i}, P_{\mathrm{c}}^{i}\right\}>61 \%$. Taking into account the corresponding membership probabilities $P_{\mathrm{t}}$ as well as structural and kinematic aspects considered in Sects. 5 and 6, the initial sample was then refined and the next approximation of $\bar{V}_{l}$ and $\bar{V}_{b}$ was computed. The iterations were repeated until the distribution parameters converged. The final value of the parameter vector was found after six iterations as $\left(\bar{V}_{l}, \bar{V}_{b}\right)=(+17.7 \pm 0.5,-11.8 \pm 0.4) \mathrm{km} \mathrm{s}^{-1}$.

In the case of an association whose size is comparable to its distance from the Sun, the proper motions of nearest and farthest association members could show significant differences. Therefore, a membership probability derived from the proper motion distribution can lead to underselection of actual members. It is exactly where we were confronted trying the membership determination from proper motions in the Per OB2 area (see Paper I). A membership selection based on tangential velocities will yield a more complete sample, although, with a higher admixture from distant background stars: due to the small peculiar velocity $\left(\bar{V}_{l}\right.$ and $\left.\bar{V}_{b}\right)$ of the association and due to increasing errors of the tangential velocities $\left(\varepsilon_{V_{l}}^{i}\right.$ and $\left.\varepsilon_{V_{b}}^{i}\right)$ with distances, background stars have "a good chance" to get larger membership probabilities $P_{\mathrm{t}}$ in Eq. (6). On the other hand, these stars can be excluded from the association sample by using spatial membership criteria and/or from their location on the CMDs. Note that a membership sample based on the proper motion criterion may miss a considerable number of association members but it is also 
not free of a contamination by field stars, although, the proportion of distant background stars is smaller.

Considering CMD criteria, we found 2065 red giants among 6172 stars brighter than $V=13$ mag which had been classified as probable members via Eq. (6). In order to estimate the fraction of field stars among the remaining 4107 main sequence stars, we analysed the distributions of their tangential velocities. For the separation of two-dimensional distribution functions, we adopted a technique based on the method of maximum likelihood (Kharchenko \& Schilbach 1995). As a result, the sample was divided into two co-moving groups with different dispersions of tangential velocities, where the group with a larger dispersion was identified to be field stars (920 stars or $22 \%$ ). Applying the same approach to the total sample of 6172 stars, we obtained a fraction of about $40 \%$ of co-moving field stars.

From the comparison of the results on the membership determination with the membership list of de Zeeuw et al. (1999), we found that out of 38 stars in common $10(24)$ are probable $\left(P_{\mathrm{t}}>61 \%\right)$ and $8(14)$ are possible $\left(1<P_{\mathrm{t}}<61 \%\right)$ members, whereas 20 (1) are field $\left(P_{\mathrm{t}}<1 \%\right)$ stars. In parenthesis we show the corresponding number of proper motion members found in Paper I. This comparison supports the result of the interloper analysis by de Zeeuw et al. (1999) who found that about $40 \%$ of their Per OB2 proper motion members might be co-moving field stars. All 6 late-type members $((B-V)>1 \mathrm{mag})$ identified by de Zeeuw et al. (1999) turned out to be field stars. Again, this result is consistent with suggestion of de Zeeuw et al. (1999) that their latetype members are possible interlopers. Out of 17 classical Per OB2 members of Blaauw (1952) we find that 5 are probable, 8 are possible members, and 4 are field stars.

\section{Spatial structure of the region}

In order to avoid biases related to the incompleteness of the data as well as to the low precision of the distance and reddening scales for red giants, we consider in this section a sample restricted to main sequence stars earlier than spectral class A7 and brighter than $V=11.6$ mag (the completeness limit of the CC). The sample, which is called hereafter MS500, provides completeness for stars earlier than A7 within about 500 pc, i.e., within the distance where the association is expected to be located, and includes 4305 stars out of $26337 \mathrm{CC}$ stars with $H_{V^{-}}$ calibrated distances. According to Eq. (6), 891 stars from MS500 are probable members of the Per OB2 association.

In Fig. 5 we show the density distribution of Per OB2 probable members in the face-on plane. The main feature of Fig. 5 is a strong correlation of the apparent density pattern with the distribution of dust clouds. It seems as if the association has a genetic relation to a "cavity" separating the Auriga (in the North) from the Taurus and Perseus (in the South) clouds. The surface density of the members ranges from 3 to 8 stars $/ \mathrm{deg}^{2}$ within this cavity whereas it is typically less than 4 stars $/ \mathrm{deg}^{2}$ in the outer

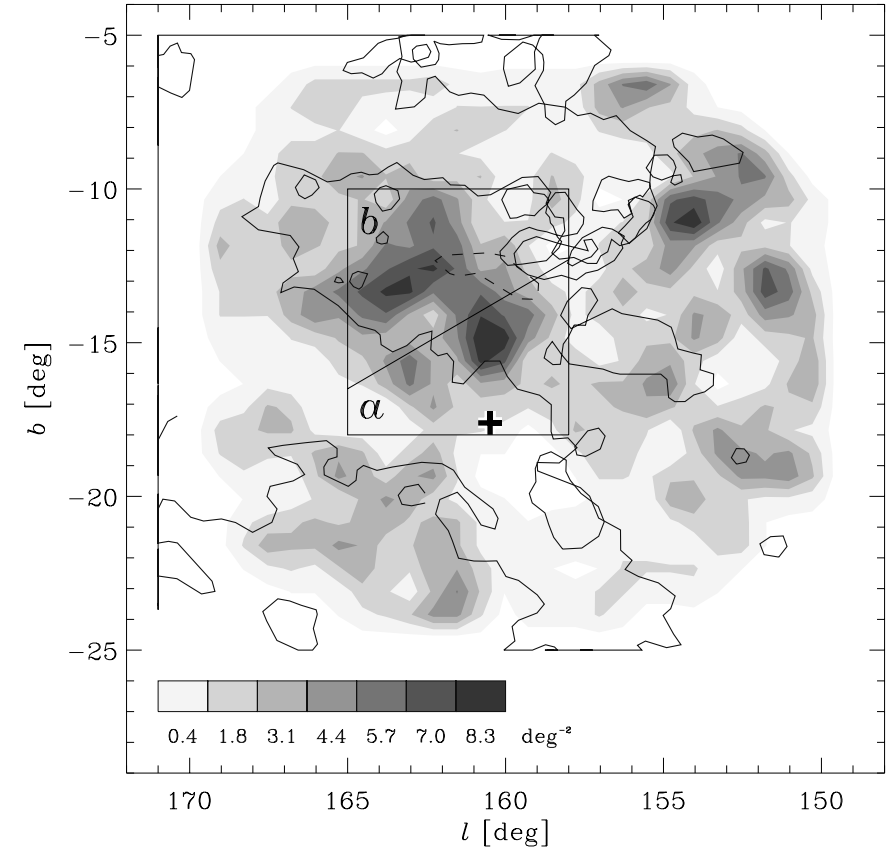

Fig. 5. The distribution of MS500 probable members $\left(P_{t}>\right.$ $61 \%)$ of the Per OB2 complex in the $(l, b)$-plane. The grey colour scale shown at the bottom of the plot indicates different levels of surface density. The solid curve outlines the CO-emitting regions related to dust clouds from the survey of Ungerechts \& Thaddeus (1987). The California nebula area is outlined by a dashed curve. The plus sign denotes the position of IC 348. The rectangular area indicated by a thin line contains the association. The Per OB2a and Per OB2b areas are separated with a line according to Eq. (7) and are marked with letters.

regions. Two peripheral areas at $(l, b)=\left(154^{\circ},-11^{\circ}\right)$ and at $\left(151^{\circ},-13^{\circ}\right)$ with an enhanced density are random concentrations of field stars, as can be concluded from their CMDs. The southern part $\left(b<-13^{\circ}\right)$ of the central concentration denoted as Per OB2a coincides with the classical position of the association (cf. Fig. 1). The northern part (Per OB2b) is bending around the California nebula which possibly hides a part of the association. Below, we discuss both regions in detail.

Although probable association members from MS500 show a clear concentration in the central part of the CC area, many of them are widely distributed across the studied field. It is reasonable to propose that the latter are background stars contaminating the sample. We can prove this assumption from the distribution of individual $H_{V}$-calibrated distances.

In Fig. 6 we show the distribution of MS500 stars with distance. The distribution of field stars rapidly increases up to a distance of about $120 \mathrm{pc}$, then the growth rate slows down, and beyond $300 \mathrm{pc}$ the distribution begins to decrease even within the assumed completeness zone. After about $600 \mathrm{pc}$ the distribution shows a long tail. The distribution of probable members is narrower due to the lack of foreground stars, and displays again a tail of distant stars. 


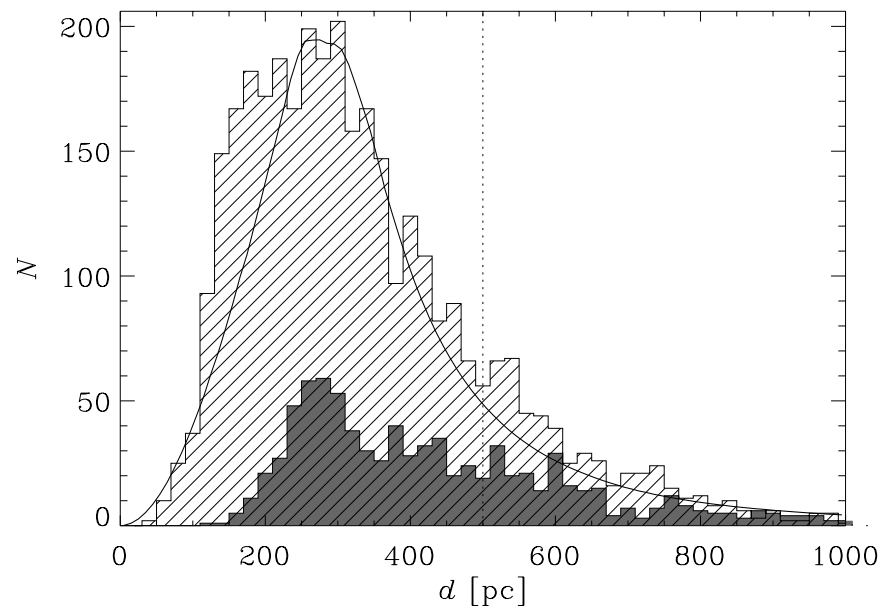

Fig. 6. Observed distribution of MS500 stars with $H_{V^{-}}$ calibrated distances. Filled histogram - probable members $\left(P_{\mathrm{t}} \geq 61 \%\right)$ of Per OB2, hatched histogram - the remaining MS500 stars $\left(P_{\mathrm{t}}<61 \%\right)$. The curve shows the model distribution of the MS stars earlier than A7, which mimics MS500 field stars. The dotted vertical line shows the completeness limit for A7V stars.

The above details can be understood either in terms of the geometry of the region or in terms of the calibration procedure used for distances. For comparison, a predicted distribution of main sequence stars earlier than A7 and brighter than $V=11.6$ is shown. It was computed in accordance with a model of galactic counts (see Kharchenko \& Schilbach 1996) for this region.

The sharp increase of the distribution at small distances simply reflects the increase of the observed spatial volume. The drop of the distribution after its maximum is a property of the magnitude limited sample of the field star population. It occurs when the fainter limit of absolute magnitudes of the stars forming the sample at a given distance passes the turning point of the field luminosity function dividing flat and steep portions of the LF ( $M_{V} \approx 4 \mathrm{mag}$ for the local LF). Then the increase of the observed space is unable to compensate for the rapidly decreasing fraction of sufficiently luminous stars, and the distribution begins to decline. The position of the maximum in Fig. 6 depends on the limiting magnitudes of the sample in apparent and absolute scales (11.6 and $2.3 \mathrm{mag}$ in our case).

The distribution of probable members peaks at about 270 pc. After the correction using Eq. (3), this corresponds to a distance to the association centre of $d_{\mathrm{a}} \approx 310 \div 330 \mathrm{pc}$. This is in a good agreement with previous results of de Zeeuw et al. (1999) who estimated the Per OB2 association distance to be within $282-318$ pc from the Sun.

The distribution of probable members does also show a long tail at larger distances. In contrast to the distribution of field MS500 stars, this bias is less caused by the limited magnitude of the sample but results from errors in the data used (see Sect. 2) and admixture of background stars. From the comparison with the distribution of probable

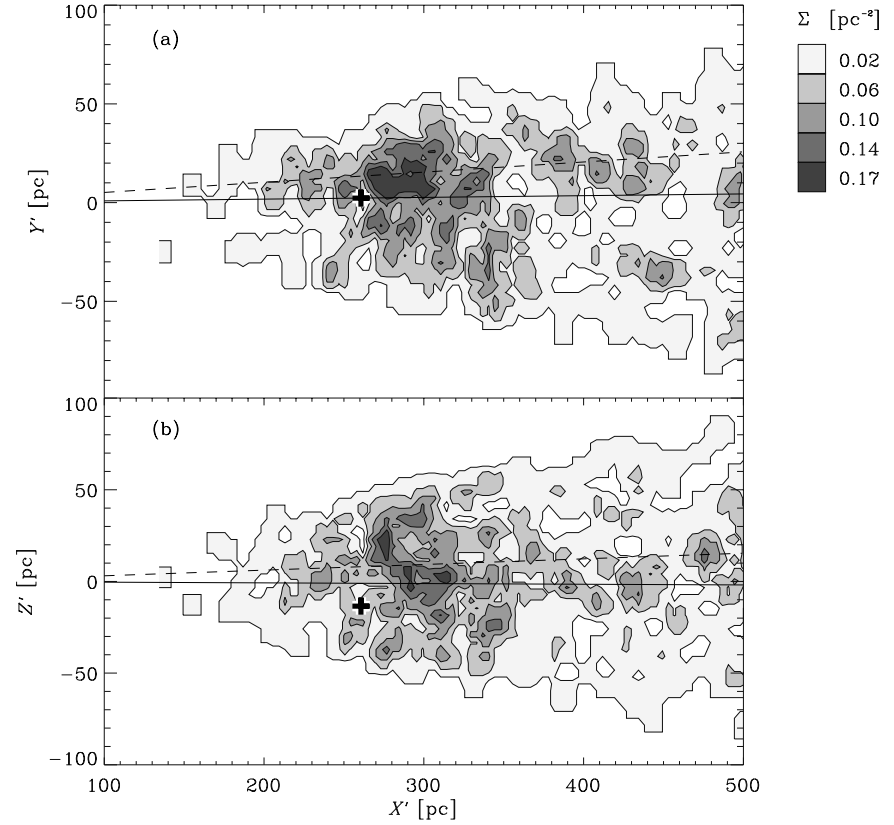

Fig. 7. The surface density of probable association members in the $\left(X^{\prime}, Y^{\prime}\right)$-plane (panel a)) and $\left(X^{\prime}, Z^{\prime}\right)$-plane (panel $\left.\left.\mathbf{b}\right)\right)$. See text for explanation. The density levels are indicated with the gray colour scale in the upper right corner of the figure. The solid lines show the axis pointed to the center of Per OB2a, the dashed lines to the center of Per OB2b. IC 348 is marked by a plus.

members selected from their proper motions, we found that the bias to larger distances is less pronounced for proper motion members. This additionally supports the assumption on the admixture of distant background stars. If compared to model calculations described in Sect. 2, the observed distribution implies a depth of the association of less than $100 \mathrm{pc}$. This is in agreement with a size of about $7^{\circ}$ for the highest concentration (i.e., a core of the association) of probable members within Per OB2ab (see Fig. 5) which corresponds to about $40 \mathrm{pc}$ at a distance of $320 \mathrm{pc}$.

In order to study the spatial distribution of stars in the Per OB2 direction, we define a proper coordinate system of the association $\left(X^{\prime}, Y^{\prime}, Z^{\prime}\right)$ related to the geometry of the region. The system is centred on the Sun and the $X^{\prime}$-axis is directed to the centre of the CC area at $(l, b)=(160.0,-14.8)$. The $Y^{\prime}$-axis points towards $(l, b)=\left(250^{\circ} 0,0.0\right)$, and the $Z^{\prime}$-axis is directed towards $(l, b)=\left(160^{\circ} 0,75^{\circ} 2\right)$. In order to produce an unbiased spatial picture, the coordinates of stars were calculated from distances, re-scaled with Eq. (3). For convenience, we call the $\left(X^{\prime}, Y^{\prime}\right)$-plane the "local equatorial projection" and the $\left(X^{\prime}, Z^{\prime}\right)$-plane the "local meridional projection". The distributions of the surface density of Per OB2 probable members in these two planes are shown in Fig. 7. The position of IC 348 in the $\left(X^{\prime}, Y^{\prime}, Z^{\prime}\right)$ coordinates is based on the distance of $261 \mathrm{pc}$ determined by Scholz et al. (1999) from Hipparcos data. 
In both planes, the regions of enhanced surface density occupy areas of approximately the same size, i.e. demonstrate a quasi-spherical shape. But their location within the CC field is non symmetric. Areas of higher density are shifted to positive $Y^{\prime}$ and $Z^{\prime}$ i.e. to the "CO-cavity" (cf. Fig. 5).

The solid and dashed lines in Fig. 7 indicate the directions to Per OB2a and Per OB2b centered at $(l, b)_{a}=$ $\left(160^{\circ} 5,-15^{\circ}\right)$ and $(l, b)_{b}=\left(163^{\circ},-13^{\circ}\right)$, respectively. As shown in Fig. 7 no separate centers could be resolved from the "local equatorial projection". An additional concentration at Per OB2a axis at $X^{\prime}=330 \mathrm{pc}$ is (according to the "meridional projection") a random superposition of regions of smaller density distributed across the CC area. In contrast, in the "meridional projection" both regions could be separated. We can identify Per OB2b in panel (b) as "northern" concentration located at $X^{\prime}=270 \div 300 \mathrm{pc}$, and Per OB2a as a region of enhanced stellar density at $X^{\prime}=280 \div 320$ pc. Then the average distance to the association is $300 \mathrm{pc}$. This distance estimation based on spatial distribution of probable association members coincides well with results derived above from the one-dimensional distribution of distances. Figure 7 also shows that the core of the association is of the order of $50 \mathrm{pc}$ in a diameter (for surface densities $\Sigma$ greater than 0.1 stars $/ \mathrm{pc}^{2}$ ), which is in agreement with data derived from the density distribution in the face-on plane (Fig. 5).

Figures 5 and 7 show that the hypothetical populations $A$ and $C$ introduced in Paper I on the basis of the analysis of proper motions and parallaxes can be identified with the Per OB2a and Per OB2b groups, respectively. From their spatial kinematics, both groups belong to the same association. Note that half a dozen de Zeeuw et al. (1999) B-type members of the association are located in the Per OB2b area, also. To prove whether Per OB2a and Per OB2b consist of populations with similar properties, we have considered the colour-magnitude diagrams for both areas.

In order to avoid sampling-dependent biases, we used a sample based primarily on spatial constraints for the construction of CMDs. We selected stars located in a rectangular area limited by the coordinates $(l, b)=\left(158^{\circ} \div\right.$ $165^{\circ},-18^{\circ} \div-10^{\circ}$ ) (see Fig. 5) considered to be the association region. We assumed that stars with

$b<82.5-0.6 \times l$,

belong to the Per OB2a area, otherwise to Per OB2b. Further, we restricted the sample to stars with $P_{\mathrm{t}}>1 \%$, $\varepsilon_{V}<0.1 \mathrm{mag}, \varepsilon_{\mu}<4 \mathrm{mas} / \mathrm{yr}$, and $d<800$ pc. For comparison, we also constructed a CMD for probable field stars located outside the association area and got membership probabilities $P_{\mathrm{t}} \leq 1 \%$. The resulting diagrams are shown in Fig. 8.

According to Fig. 8, both association regions show a remarkable similarity in their CMD properties. Both Per OB2a and Per OB2b samples demonstrate clear main sequences and the same degree of contamination by field

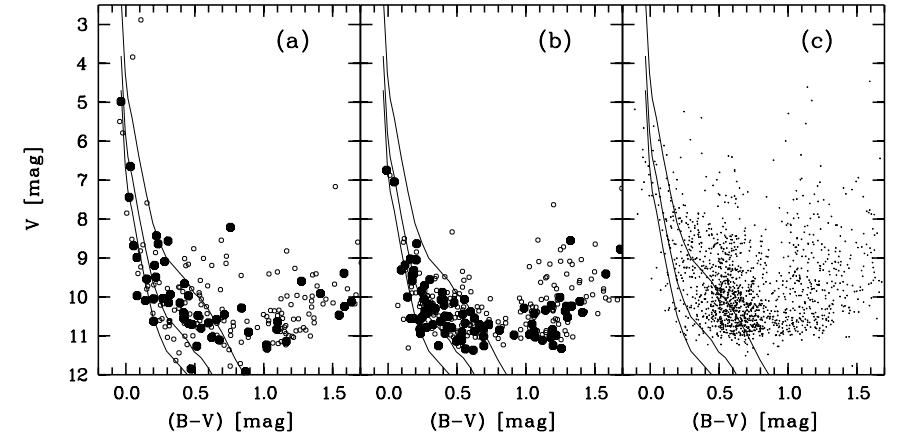

Fig. 8. Colour magnitude diagrams of Per OB2a (panel a), Per OB2b (panel b) and outer (panel c) regions of the CC. Large filled circles mark the probable members of the association $\left(P_{t}>61 \%\right)$, small open circles - the possible members $\left(P_{t}>1 \%\right)$, and dots - the probable field stars $\left(P_{t} \leq 1 \%\right)$. Curves are ZAMS of Schmidt-Kaler (1982) reddened by average extinction values $\left(A_{V}=1 \mathrm{mag}\right.$, and $\left.E_{(B-V)}=0.3 \mathrm{mag}\right)$, for stars located at 200,400, and $600 \mathrm{pc}$ (from right to left).

red giants (stars with $(B-V)>1 \mathrm{mag}$ ). The only difference between both diagrams is the presence of five very bright $(V<6 \mathrm{mag})$ stars in Per OB2a, the evident reason why it was identified as an association in the first place. As the positions of the empirical ZAMS show, both groups are located at a distance between 200 and $400 \mathrm{pc}$ which is in agreement with previous findings. Both diagrams are in strong contrast (if one does not take into account red giants) with the field star CMD which show a vertical sequence at $(B-V) \approx 0.5 \mathrm{mag}$ of main sequence stars at different distances. Thus, we conclude that the Per OB2a and Per OB2b regions represent the same population both from kinematic and CMD points of view. Consequently, the Per OB2 association is a spatially more extended group, in contrast to the classical results considering the association to be mainly located in the Per OB2a area.

\section{Kinematics of the region}

Once the area occupied by the association is outlined and its members are selected, the study of its spatial motions (both motion of the association as a whole, and internal systematic motions like rotation or expansion/contraction) becomes possible.

The hypothesis on expansive motions in OBassociations was introduced in the early work of Ambarzumian (1949). Later this problem was studied in detail by Blaauw. The expansion of Per OB2 was examined by Blaauw (1952), Delhaye \& Blaauw (1953), and Lesch (1969) from proper motions and radial velocities of the brightest members of the association and they found some evidence of its expansion. According to their results, the kinematic age of the association inferred from the measured expansion rate is about 1.3 Myr.

In order to get an unbiased picture of the complex internal motions, the kinematic data were corrected for 


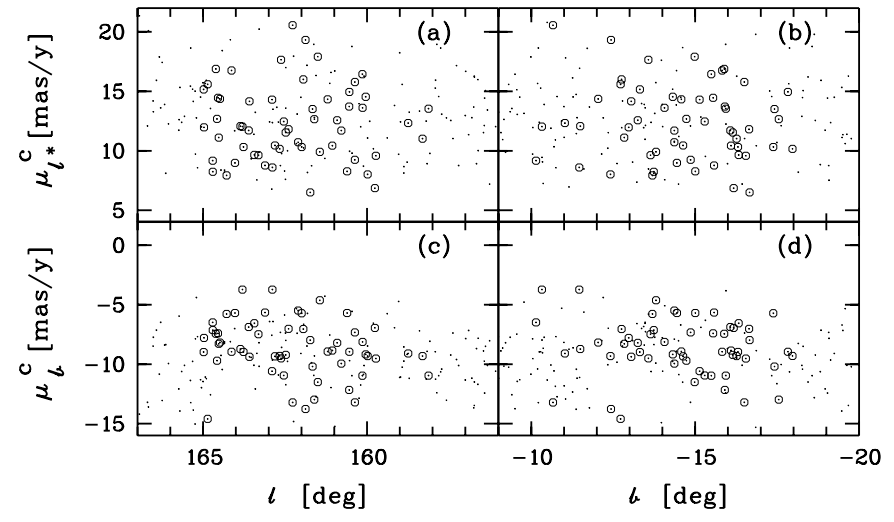

Fig. 9. Corrected proper motions of probable members of Per OB2 versus Galactic coordinates. Dots - stars from the complete field of the CC, circles - stars from the central (association) area (= KAS).

the Solar motion and differential rotation of the Galactic disk as described in Sect. 4. Only stars with accurate proper motions $\left(\varepsilon_{\mu}<3 \mathrm{mas} / \mathrm{yr}\right)$ were used for the evaluation of systematic motions. Among probable association members, we selected main sequence stars brighter than $V=11.6 \mathrm{mag}$ and with distances between 200 and $400 \mathrm{pc}$. Totally, 276 stars were found in the CC to fit the criteria. These stars are shown by dots in Fig. 9. A subsample of 57 stars located in the Per OB2ab area and called hereafter the Kinematic Association Sample (KAS) is marked by open circles in Fig. 9 .

In the case of a significant internal radial motion, it should be revealed from $\left(\mu_{l^{*}}^{\mathrm{c}}, l\right)$ - and $\left(\mu_{b}^{\mathrm{c}}, b\right)$-diagrams (panels (a) and (d) in Fig. 9), whereas the rotation of the association around the line of sight would be indicated in $\left(\mu_{l^{*}}^{\mathrm{c}}, b\right)$ - and $\left(\mu_{b}^{\mathrm{c}}, l\right)$-planes (panels (b) and (c)). No significant evidence of systematic internal motions in the association can be inferred from Fig. 9.

This conclusion is supported by a linear least square solution of individual motions of KAS stars yielding:

$\mu_{l^{*}}^{\mathrm{c}}=(+0.04 \pm 0.24)(l-160)^{\circ}+(12.2 \pm 0.7)$,

$\mu_{b}^{\mathrm{c}}=(+0.18 \pm 0.17)(b+15)^{\circ}+(-8.7 \pm 0.3)$,

$\mu_{l^{*}}^{\mathrm{c}}=(+0.22 \pm 0.23)(b+15)^{\circ}+(12.2 \pm 0.4)$,

$\mu_{b}^{\mathrm{c}}=(+0.21 \pm 0.18)(l-160)^{\circ}+(-9.4 \pm 0.5)$.

Here $\mu_{l^{*}}^{\mathrm{c}}$ and $\mu_{b}^{\mathrm{c}}$ are proper motions corrected for the Solar motion and differential rotation of the Galactic disk. The angular coefficients in the equations are not significant. We conclude that within the accuracy of the present data, no systematic internal motions are detectable in the Per OB2 association.

Probably, the previous results indicating an expansion of the Per OB2 are biased due to insufficiently accurate proper motions and membership selection and/or correction of velocities for the differential rotation of the Galactic disk and Solar motion. This assumption is supported by extensive simulations by Brown et al. (1997) who pointed at the importance of all these factors in the determination of internal motions and kinematic ages of associations. These authors also concluded that even for nearby associations, the Hipparcos accuracy is not sufficient to derive these parameters properly.

Using the KAS stars, we derived various estimators of the motion of the association. The averaged proper motion, not corrected for the systematic effects, was obtained as $\left(\bar{\mu}_{l^{*}}^{\mathrm{O}}, \bar{\mu}_{b}^{\mathrm{O}}\right)=(+10.9 \pm 0.4,-2.1 \pm 0.2) \mathrm{mas} / \mathrm{yr}$. This agrees well with the results derived by de Zeeuw et al. (1999) for Per OB2: $(+8.4 \pm 0.2,-2.3 \pm 0.3) \mathrm{mas} / \mathrm{yr}$. The averaged tangential velocity of the association with respect to the LSR computed from proper motions corrected for the parallactic motion of the Sun is $\left(\bar{V}_{l}, \bar{V}_{b}\right)=$ $(+7.2 \pm 0.4,+2.0 \pm 0.3) \mathrm{km} \mathrm{s}^{-1}$. For nine KAS stars, radial velocities are available in the $\mathrm{CC}$. The averaged radial motion of these stars is $\bar{V}_{R}=+20.0 \pm 1.4 \mathrm{~km} \mathrm{~s}^{-1}$. The spatial velocity components $(U, V, W)$ of the Per OB2 association with respect to the LSR were computed from the averaged tangential and radial velocities as $(U, V, W)=$ $(+12.7 \pm 1.6,-3.0 \pm 0.6,-0.9 \pm 0.8) \mathrm{km} \mathrm{s}^{-1}$.

\section{Discussion and conclusions}

In this paper we deal with one of the nearest associations with a long history of scientific investigations, the Per OB2 association. On one hand, this association is predestined for membership studies: due to a rather high Galactic latitude $\left(b \approx-15^{\circ}\right)$ it is projected onto a relatively poor background of the thick disk. On the other hand, its average velocity does not differ strongly from that of the field stars. Therefore, the full potential of the usage of the proper motion method for membership determination cannot be exploited.

For our study we applied a deeper and more complete data set of present-day quality in the region of Per OB2 than was used before. We implemented the reduced proper motion method, which together with the high accuracy of the kinematic data, proved to be a very effective tool for the study of the association. Compared to previous investigations, we probed a much larger sample of 30000 stars with accurate proper motions in the Hipparcos system and magnitudes being updated with other relevant data.

Since proper motions are the most common type of data within our sample, we combined these data with available spectral classification and used them for distance calibration. In spite of the unfavourable conditions for membership determination, this enabled us to select the association members with higher reliability. Monte Carlo simulations show that due to the still insufficiently accurate proper motions and due to the non-zero internal random motions of the association stars, we can hardly study the association structure in detail from individual $H_{V^{-}}$ calibrated distances. However, we can apply the method for an independent and robust determination of the distance to the association and for the separation of main sequence stars from red giants.

The extensive sample of spectral classification data included in the $\mathrm{CC}$ allowed to study the distribution 
of interstellar extinction in the wider neighbourhood of the association. We found a remarkable correlation of the reddening pattern with the structure of CO-emitting clouds derived from large scale radio-surveys. In the studied region, the average reddening was determined to be $\bar{E}_{(B-V)}=0.31 \mathrm{mag}$, and $\bar{A}_{V}=1.0 \mathrm{mag}$.

With distances estimated practically for every star in the CC, we had some freedom to decide which approach to use for the member selection (proper motions or tangential velocities). In the case of extended structures like associations, a membership criterion based on tangential velocities is more justified. However, we found that, at the present accuracy level of the data, both approaches give, in general, the same association structure, being somewhat different only for distance-dependent features. The implementation of a membership criterion based on tangential velocities to all CC stars brighter than $V=13$ mag yielded about 6200 probable members (i.e., membership probability $P_{\mathrm{t}}>61 \%$ ). However, the analysis of their distribution over the sky and their CMDs have shown that this sample is strongly contaminated by co-moving field stars. When applied to a uniform sample of main sequence stars located within 500 pc from the Sun, the kinematic criterion has revealed a clear concentration (i.e. the association core) in the central part of the $\mathrm{CC}$ field with a size of about $7^{\circ}$.

In this region, we found 1025 probable main sequence members with $V$ between 3 and 13. Assuming a 20\%contamination by field stars (see Sect. 4), this amounts to about 800 association members within a mass range of $m=1-17 m_{\odot}$. Under the assumption that the Per OB2 population goes down to masses of $0.1 m_{\odot}$, we estimate adopting the Salpeter IMF - that these stars would make up about $4 \%$ of the total number of association members (i.e., 20000 members with an average mass of $0.32 \mathrm{~m}_{\odot}$ ). With a core radius of $25-20 \mathrm{pc}$, this yields an average density of about $0.1-0.2 m_{\odot} / \mathrm{pc}^{3}$. This estimation is in agreement both with mass densities expected for associations (0.001-0.1 $\mathrm{m}_{\odot} / \mathrm{pc}^{3}$, see Lang 1992) and with the average densities of open clusters like the Pleiades $\left(0.18 m_{\odot} / \mathrm{pc}^{3}\right)$ or Praesepe $\left(0.25 m_{\odot} / \mathrm{pc}^{3}\right)$ obtained by Kholopov (1981).

The analysis based on these stars also shows that the previous view of Per OB2 should be modified by extending the association in the direction of the Galactic equator, towards the California nebula and the Auriga dark cloud. This brings the association in full coherence with the environment, dominated by the cloud pattern. We conclude that the Per OB2 association is not only in relation with the Perseus cloud in the South, but also interacts with the Auriga cloud in the North. It cannot be excluded that association stars are responsible for the "cavity" separating the Auriga and Taurus/Perseus cloud systems.

The distance to the association center derived from kinematic calibration is found to be about $300 \mathrm{pc}$. This is in agreement with Hipparcos-based data of de Zeeuw et al. (1999) found for about 40 members. Taking also into account the estimated diameter of the association core of about $40 \mathrm{pc}$, we conclude that the IC 348 cluster which is semi-embedded into the Perseus dark cloud is located immediately at the front edge of the association. The derived results support our findings from Paper I that the stellar population of Per OB2 consists of two groups located at different distances. According to our new results, these groups called Per OB2a and Per OB2b which represent the same kinematic sample and show similar CMDs but are separated in the face-on plane and along the line of sight by a few tens of parsecs, can be identified with these two populations found previously.

This configuration may lead to an interesting consequence. Indeed, as we note in Sect. 5, the only difference between the CMDs of the Per OB2a and Per OB2b regions is the lack of the luminous stars in the latter. If one interprets this as an age difference, then it can not be ruled out, that the Per OB2 association represents an example of propagated star formation, which started in Per OB2b at the edge of the Auriga clouds more than $30 \mathrm{Myr}$ ago, resumed in Per OB2a after about $10 \mathrm{Myr}$, and is now in progress at the Per OB2 southern border within the Perseus cloud (IC 348). We plan to consider this issue in a following paper.

The examination of internal systematic motions has shown that no evidence of an expansion of the association nor a rotation around the line of sight is found from the proper motion data. Radial velocities are too scarce and inaccurate to produce a coherent picture of internal motions. However they are sufficient to be involved in the calculation of the mean spatial motion of the association.

Acknowledgements. This work is supported in part by the German Deutsche Forschungsgemeinschaft, DFG project number $113 / 380 / \mathrm{O}(\mathrm{R})$ and by the Russian Foundation of Basic Research (grant No. 01-02-16306). RDS gratefully acknowledges financial support from the Deutsches Zentrum für Luftund Raumfahrt (DLR) (Förderkennzeichen 50 OI 0001). We acknowledge the use of the SIMBAD database operated at the CDS. The authors also thank the anonymous referee for his/her very useful comments and suggestions.

\section{References}

Ambarzumian, V. A. 1949, Astron. Zhurn., 26, 3

Belikov, A. N., Kharchenko, N. V., Piskunov, A. E., et al. 2002, A\&A, 384, 145 (Paper I)

Blaauw, A. 1952, BAN, 11, 405

Blaauw, A. 1964, ARA\&A, 2, 213

Brown, A. G. A., Dekker G., \& de Zeeuw, P. T. 1997, MNRAS, 285,479

Černis, K. 1990, Ap\&SS, 166, 315

Černis, K. 1993, Baltic Astr., 2, 214

Chiu, L.-T.G. 1980, ApJS, 44, 31

Dehnen, W., \& Binney, J. J. 1998, MNRAS, 298, 387

Delhaye, J., \& Blaauw, A. 1953, BAN, 12, 72

Efremov, Yu. N. 1981, Star formation sites in galaxies. Star complexes and spiral arms (Nauka Publ., Moscow)

Feast, M. W., \& Whitelock, P. 1997, MNRAS, 291, 683

Houk, N., Swift, C. M., Murray, C. A., Penston, M. J., \& Binney, J. J. 1997, ESA SP-402, 279 
Jones 1972, ApJ, 173, 671

Kharchenko, N., \& Schilbach, E. 1995, Astron. Nachr., 1995, 316,91

Kharchenko, N., \& Schilbach, E. 1996, Baltic Astr., 5, 337

Kislyuk, V., Yatsenko, A., Ivanov, G., Pakulyak, L., \& Sergeeva, T. 1999, in Motion of Celestial Bodies, Astrometry and Astronomical Reference Frames, JOURNEES 1999 \& IX Lohrman Kolloquium (Dresden, Germany), 13-15 September 1999

Kholopov, P. N. 1981, Star Clusters (Nauka Publ., Moscow.)

Lang, K. R. 1992, Astrophysical Data: Planets and Stars (Springer-Verlag, New York, Berlin, Heidelberg, London, Paris, Tokyo, Honk Kong, Barcelona, Budapest)

Lesh, J. R. 1969, AJ, 74, 891
Sakhibov, F., \& Smirnov, M. A. 2000, A\&A, 354, 802

Schmidt-Kaler, Th. 1982, Landolt-Börnstein Numerical Data and Functional Relationships in Science and Technology, New Series, Group IV (Springer-Verlag Press, BerlinHeidelberg, New York), 2, 15

Scholz, R.-D., Brunzendorf, J., Ivanov, G., et al. 1999, A\&AS, 137, 305

Seyfert, C. K., Hardie, R. H., \& Grenchik, R. T. 1960, ApJ, 132,58

Straižys, V. 1977, Multicolour stellar photometry (Mokslas Publ., Vilnius)

Ungerechts, H., \& Thaddeus, P. 1987, ApJS, 63, 645

de Zeeuw, P. T., Hoogerwerf, R., de Bruijne, J. H. J., Brown, A. G. A., \& Blaauw, A. 1999, AJ, 117, 354 